\title{
CRITICAL INITIAL CONDITIONS FOR SPATIALLY-DISTRIBUTED THERMAL EXPLOSIONS
}

\author{
A. A. LACEY ${ }^{1}$ and G. C. WAKE ${ }^{2}$
}

(Received 21 January 1991; revised 3 April 1991)

\begin{abstract}
The problem of finding critical initial data which separate conditions leading to blow-up from those which give solutions tending to the (stable) minimal solution is considered. New criteria for blow-up and global existence are found; these are equivalent to obtaining upper and lower bounds respectively for the set of critical initial data.
\end{abstract}

\section{Introduction}

Semi-linear parabolic equations of the form

$$
u_{t}=\Delta u+f(u),
$$

are well known to be capable of exhibiting blow-up for functions $f$ which grow sufficiently fast with $u$. By this we mean that if we consider the initialboundary value problem $(P)$, which is (1) in some open bounded region $\Omega$ in $\mathbf{R}^{N}, t>0$, with the (linear) boundary condition

$$
B u \equiv \alpha u_{n}+(1-\alpha) u=0, \quad \text { on } \partial \Omega, t>0
$$

where we assume $\partial \Omega$ is smooth, $0 \leq \alpha(x) \leq 1, u_{n}$ is the outer normal derivative on $\partial \Omega$, and the initial condition is

$$
u(x, 0)=\phi(x) \quad \text { in } \bar{\Omega} \text { at } t=0,
$$

\footnotetext{
${ }^{1}$ Mathematics Department, Heriot-Watt University, Edinburgh EH14 4AS, Scotland.

${ }^{2}$ Department of Mathematics, Massey University, Palmerston, North, New Zealand.

(C) Copyright Australian Mathematical Society 1992, Serial-fee code 0334-2700/92
} 
then the solution $u(x, t)$ is unbounded above as $t \rightarrow t^{*}<\infty$. We call $t^{*}$ the "blow-up time".

Throughout this paper we shall be considering (1) as modelling an exothermic chemical reaction, with $u$ representing temperature and $f$ being essentially the temperature-dependent reaction rate. The representation by a single equation, so that reactant depletion is neglected, is valid provided that $u$ does not get too large. In this case the reaction rate which is more accurately modelled by the Arrhenius law $f(u)=\delta \exp (u /(1+\varepsilon u))$ can be approximated by an exponential function (the Frank-Kamenetskii approximation) $f(u)=\delta e^{u}$, for some $\delta>0$. (See Boddington et al. [4]).

For such problems, where $f(s), f^{\prime}(s), f^{\prime \prime}(s)$ are all positive for $s \geq 0$ and

$$
\int_{0}^{\infty} d s / f(s)<\infty
$$

it is known (see Keller and Cohen [8]) that for region $\Omega, \delta$, or $\alpha$ sufficiently large then there is no steady-state solution of (1), (2). We then have the result (see Lacey [9]; Bellout [3]) that the solution to (1), (2), (3) blows up for any initial data $\phi(x) \geq 0$ (or any $\phi$ positive or negative if $f(s)>0$ for all $s \in \mathbf{R})$. In this case there is ignition for the physical system being modelled. As the temperature $u$ becomes large, we say that thermal runaway occurs.

In many applications, the region and boundary conditions are such that steady-states are possible, but given sufficiently large initial data, the system still blows up. Some practical cases of interest with initial data high enough to cause the material to ignite are described in Gray and Scott [6]. The same paper gives some numerically computed values of uniform critical initial data for balls in 1,2,3 dimensions. The question of whether or not a system will ignite with a given initial condition is of high practical interest. We wish to improve on both necessary and sufficient conditions for blow-up. This is the first principal aim of this paper. In Section 2, we review briefly the known criteria for blow-up and global existence, which is followed in Section 3 by a new method of obtaining bounds for critical initial conditions. The other intention, recognising that for many problems numerical solution is going to be important for discovery of whether or not blow-up occurs, will be to describe the structure of the set of critical initial conditions; that is, those functions $\phi(x)$ which neither blow up nor tend to the minimal steady-state (the only one which can be stable). The aim here is to allow deductions to be drawn on solutions of the initial value problem (1), (2), (3), given knowledge (perhaps obtained numerically) for somewhat different problems. This is done in Section 4. 


\section{Known conditions on initial conditions for blow-up}

We now suppose there is a steady-state for (1) and (2), and we shall seek those functions $\phi$ which lead to blow-up and those which do not.

\section{A. (Energy method)}

One class of methods is based on energy, and is most suitable for problems where $f(u)=u^{1+b}, b>0$ (or something similar); of course for this we must relax one requirement on $f$ so that " $f(s)>0$ for $s \geq 0$ ". Following Ball [1] and Wake and Rayner [15], we define (for $\alpha$ constant or zero)

$$
E=\int_{\Omega}\left(\frac{1}{2}|\nabla u|^{2}-F(u)\right) d x+\frac{(1-\alpha)}{2 \alpha} \int_{X \in \Gamma} u^{2} d s(x),
$$

where $\Gamma=\{x \in \partial \Omega: \alpha>0\}$ and $F(u)=\int_{0}^{u} f(s) d s$. By differentiating (5) with respect to $t$, we get

$$
\dot{E} \equiv \frac{d E}{d t}=\int_{\Omega}\left(\nabla u \cdot \nabla u_{t}-F^{\prime}(u) u_{t}\right) d x+\frac{1-\alpha}{\alpha} \int_{\Gamma} u u_{t} d s(x) .
$$

Using the divergence theorem, and (1), (2), we obtain

$$
\dot{E}=-\int_{\Omega} u_{t}^{2} d x
$$

and so $\dot{E} \leq 0$ ( $\dot{E}<0$ unless $u$ is a steady-state), and $E(t) \leq E_{0} \equiv E(0)$. Defining $J=\int_{\Omega} u^{2} d x$, we obtain, using (1),

$$
\begin{aligned}
\dot{J} & =2 \int_{\Omega}(u \Delta u+u f(u)) d x \\
& =2 \int_{\Omega}\left(u f(u)-|\nabla u|^{2}\right) d x+2 \int_{x \in \partial \Omega} u u_{n} d s(x) \\
\dot{J} & =2 \int_{\Omega}(u f(u)-2 F(u)) d x-4 E+2 \int_{x \in \partial \Omega} u\left(u_{n}+\frac{1-\alpha}{\alpha} u\right) d s(x) .
\end{aligned}
$$

In view of the boundary condition (2) and noting that $u(x)=0$ on $\partial \Omega \backslash \Gamma$, the boundary term in the last equation vanishes. Accordingly

$$
\dot{J} \geq 2 \int_{\Omega}(u f(u)-2 F(u)) d x-4 E_{0} .
$$

Further, if $E_{0} \leq 0$, we have

$$
\dot{J} \geq 2 \int_{\Omega}(u f(u)-2 F(u)) d x .
$$

In the case of $f(u)=u^{1+b}, b>0,(5)$ gives, using Jensen's inequality,

$$
\dot{J} \geq \frac{2 b}{2+b} \int_{\Omega} u^{2+b} d x \geq C J^{(2+b) / 2}
$$


where $C$ is independent of $u$ and so $J$ becomes unbounded after a finite time (less than $\left.(2 / b C) J_{0}^{-b / 2}\right)$, under the assumption that a solution continues to exist. We conclude that blow-up must occur.

For more general $f$, in particular with $f(0)=A>0$, we have for the expression on the right-hand side of the inequality (6)

$$
\int_{\Omega}(u f(u)-2 F(u)) d x=\int_{\Omega} h(u) d x-A \int_{\Omega} u d x,
$$

where $h(u)=u(f(u)+A)-2 F(u)$ is a positive increasing function (and $h(u) / u^{2} \rightarrow \infty$ as $\left.u \rightarrow \infty\right)$. Then, again in the equality (6)

$$
j \geq C h\left(J^{1 / 2}\right)-c A J^{1 / 2},
$$

for some $C>0$ and $c=(\text { volume of } \Omega)^{1 / 2}$. This gives blow-up provided that

$$
\int_{1}^{\infty} d s / h\left(s^{1 / 2}\right)<\infty \text { and } J(0) \text { is sufficiently large . }
$$

Again if we denote $W=\{v \in C(\Omega): v$ is a steady state of (1), (2)\}, then if

$$
E_{0}<\min _{v \in W} E(v),
$$

since $E$ is decreasing in time, then $u$ does not tend to a steady-state and must therefore be unbounded. But if $E \rightarrow-\infty$ we must have $J \rightarrow \infty$ and we again deduce blow-up.

\section{B. (Bounding solutions)}

Following Lacey [9], we take some $g(x) \geq 0, g \neq 0$ and (support of $g) \cap(\partial \Omega \backslash \Gamma)$ empty, and define $z$ so that

$$
\Delta z+g=0, \quad x \in \Omega,
$$

together with the boundary condition (2). Then we may take the constant a large enough such that

$$
a \Delta z+f(a z) \geq 0 .
$$

If $\phi \geq a z$, the solution to the initial value problem (1), (2), (3) will blow-up.

C. (Fourier coefficient method, see Kaplan [7]; Tzanetis [13])

Taking $\lambda, \phi$ to be the principal eigen-pair for the Helmholtz problem

$$
\begin{aligned}
\Delta \psi+\lambda \psi & =0, & & x \in \Omega, \\
B(\psi) & =0, & & x \in \partial \Omega,
\end{aligned}
$$

and scaling so that $\int_{\Omega} \psi d x=1$, then if $a(t)$ is the Fourier coefficient

$$
a(t)=\int_{\Omega} u(x, t) \psi(x) d x
$$




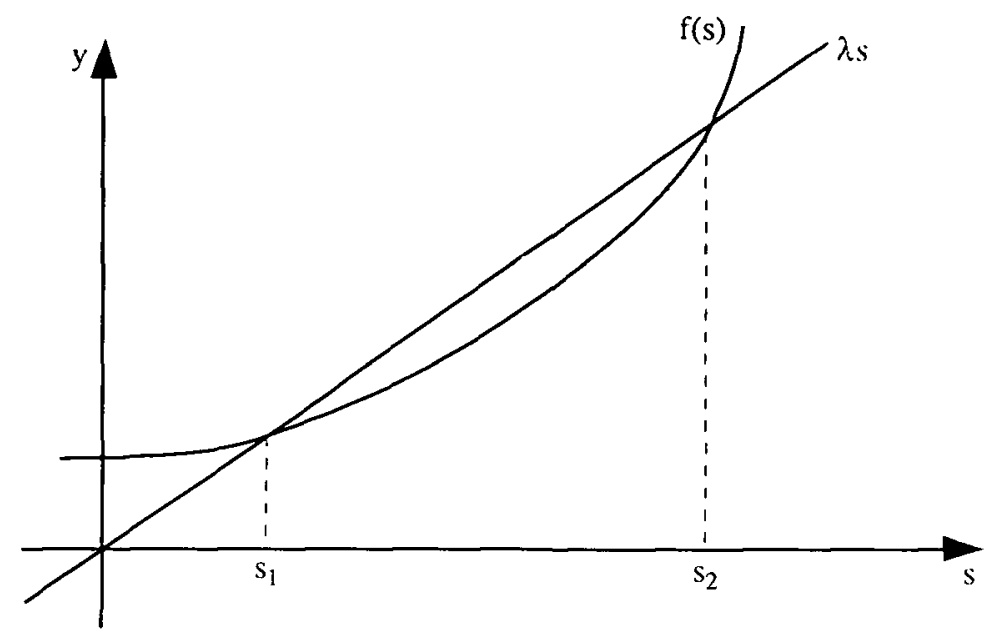

FIGURE 1. Schematic diagram of graphs showing calculation of criteria for blow-up.

we have

$$
\dot{a}=\int_{\Omega} \psi(\Delta u+f(u)) d x=\int_{\Omega}(f(u)-\lambda u) \psi d x,
$$

or

$$
\dot{a} \geq f(a)-\lambda a .
$$

For $f$ of the required form (satisfying the conditions (4)) and under the assumption of existence of a steady-state, $f(s)=\lambda s$ has two roots $s_{1}$, $s_{2}\left(s_{1}<s_{2}\right)$, between which $f(s)<\lambda s$ and outside of which $f(s)>\lambda s$ (see Figure 1).

Again, if $a(0)=\int_{\Omega} \phi(x) \psi(x) d x \geq s_{2}$ we get blow-up.

D. (Fourier coefficients method, see Lacey [9], Sattinger [12], Fujita [5])

If there are at least two steady states then one of them, say $w$, is minimal and stable. We denote a second steady-state as $v$ with $v>w$, and $v$ is unstable since the principal eigenvalue for $\Delta \psi+f^{\prime}(v) \psi=\lambda \psi$ in $\Omega$, with the boundary condition (2), is positive. We scale $\psi$ so that $\int_{\Omega} \psi d x=1$. Defining now

$$
a(t)=\int_{\Omega} \psi(x)(u(x, t)-v(x)) d x,
$$

we obtain

$$
\dot{a} \geq h(a)+\lambda a,
$$

where $h$ is some function satisfying $h(0) \geq 0 ; h(s)>0, s \neq 0 ; h^{\prime}(s)>0$, $h^{\prime \prime}(s)>0$ for $s>0 ;$ and $\int_{1}^{\infty} d s / h(s)<\infty$. The inequality (7) for $\dot{a}$ is strict except when $u \equiv v$. Then we conclude that if $a(0) \geq 0$ and $\phi \not \equiv v, u$ must blow up. Also if $\phi(x) \leq v(x), \phi \not \equiv v$, then $u \rightarrow w$ as $t \rightarrow \infty$ (see for example, Sattinger [12]). 
In this case, the nonminimal steady-state is a critical initial condition i.e. if $\phi<v, u \rightarrow w$; if $\phi>v, u$ blows up.

We now wish to use this last method as a basis for more conditions. In particular the only criteria so far for a global solution has been that $\phi \leq v$ and we shall now find some initial conditions which lead to $u$ tending to $w$ even though $\phi(x)>v(x)$ for some $x \in \Omega$. This will enable us to obtain better estimates for what is termed the SET OF CRITICAL INITIAL CONDITIONS. To completely characterise this set is clearly impossible.

\section{New conditions for global existence or blow-up}

We take a one-parameter family of functions $f(u, \beta)$ such that $f_{\beta} \geq 0$, $f(\cdot, 0)=f$ and an increasing function $g(\beta)$ such that $g(0)=0$, and for any $\beta \neq 0$, at least one of

$$
(f(\cdot ; \beta)-f(\cdot)), g(\beta) \neq 0 .
$$

The function $z(x ; \beta)$ is defined to be the solution of

$$
\left.\begin{array}{rl}
\Delta z+f(z ; \beta)=0 & \text { in } \Omega, \\
B z=g(\beta) & \text { on } \partial \Omega .
\end{array}\right\}
$$

If there is more than one solution, we take $z$ to be a nonminimal solution (in order to get stronger results). We note that in general $\left(P_{\beta}\right)$ has solutions for some $\beta>0$ as well as $\beta \leq 0$. However, for the special critical case where

$$
\begin{gathered}
\Delta V+\lambda f(V)=0 \quad \text { in } \Omega, \\
B V=0 \text { on } \partial \Omega,
\end{gathered}
$$

fails to have solutions for $\lambda>1$, there will not be solutions to $\left(P_{\beta}\right)$ for $\beta$ positive.

Let us now suppose there are two steady-state solutions for (1), (2), $v$ and $w$, with $v>w$ and $w$ the minimal solution. Take $z$ to be a nonminimal solution of $\left(P_{\beta}\right)$ with $\beta<0$. Since $\beta<0, f_{\beta} \geq 0$, and in view of the conditions in (8), it follows that

$$
\Delta z+f(z) \geq 0 \quad \text { in } \Omega, \quad B(z) \leq 0 \quad \text { on } \partial \Omega,
$$

with at least one inequality being strict, so that $z$ is a strict lower solution for (1) and (2).

It follows that if $\phi(x) \geq z(x)$ on $\Omega$, then $u$ blows up. (We note that $z(x)<w(x)$ on parts of the region $\omega$.) 
Conversely, if the system is subcritical, we may take some $\beta>0$ and $z$ the solution (preferably not the minimal solution, so as to get a higher bound) to $\left(P_{\beta}\right)$. Now

$$
\Delta z+f(z) \leq 0 \quad \text { in } \Omega, \quad B(z) \geq 0 \quad \text { on } \partial \Omega
$$

with at least one inequality being strict, so that $z$ is a strict upper solution for (1) and (2). It follows that if $\phi(x) \leq z(x)$ then $u$ is bounded and must tend to a steady-state. Now, taking $\phi=z$ we see that since blow-up does not occur, $z<v$ somewhere (assuming that a second steady-state exists) and $u$ is also monotonic decreasing with time $t$. We conclude that $u$ tends to the minimal steady-state.

We have above a way of constructing upper and lower bounds to the set of critical initial conditions, which uses solutions of related steady-state problems. We now consider an example in which we are able to use explicit solutions of the steady-state problem, but of course, even for situations where explicit solutions are not known, the method works equally well using solutions determined by numerical methods.

EXAMPLE. Take $\Omega=B(0,1) \subseteq R^{2}$, the two-dimensional unit ball, with $f(u)=e^{u}$, and, for simplicity $\alpha=0$, that is, we have Dirichlet conditions. The steady-state solutions to (1) satisfy

$$
u_{r r}+u_{r} / r+e^{u}=0, \quad 0<r<1 ; \quad u_{r}(0)=0
$$

and are given by $u=-2 \ln \left(a+b r^{2}\right)$, with $8 a b=1$. Taking $f(u ; \beta)=$ $f(u)=e^{u}$ and $g(\beta)=\beta$, the boundary condition gives

$$
-2 \ln (a+b)=\beta \quad \text { or } \quad b=e^{-\beta / 2}-a .
$$

The steady-states to (1), (2) (that is, $\beta=0$ ) thus satisfy $b=1-a$, $8 a(1-a)=1$ and $a=1 / 2(1 \pm 1 / \sqrt{2})$. Hence

$$
\begin{aligned}
& v=\ln 8-2 \ln \left\{\sqrt{2}+1+(\sqrt{2}-1) r^{2}\right\}, \\
& v=\ln 8-2 \ln \left\{\sqrt{2}-1+(\sqrt{2}+1) r^{2}\right\}
\end{aligned}
$$

are the steady-states.

Firstly, taking $\beta<0, z$ is given by $8 a\left(e^{-\beta / 2}-a\right)=1$ or $a=$ $\frac{1}{2}\left\{e^{-\beta / 2}-\left(e^{-\beta}-\frac{1}{2}\right)^{1 / 2}\right\}$ (for the nonminimal solution we need the smaller value of $a$ ). Thus by the results above, for all $\beta \leq 0$

$$
\begin{aligned}
\phi(r) \geq z_{1}=\ln 8-2 \ln \left\{\sqrt{2} e^{-\beta / 2}\right. & -\left(2 e^{-\beta}-1\right)^{1 / 2} \\
& \left.+\left(\sqrt{2} e^{-\beta / 2}+\left(2 e^{-\beta}-1\right)^{1 / 2}\right) r^{2}\right\}
\end{aligned}
$$


ensures blow-up will occur. We note that near the boundary, where $z_{1}=$ $\beta<0$, the comparison function $z$ is less than $v$ and thus gives a different sufficient condition for blow-up, or conversely a necessary condition for global existence to $(P)$. Secondly, taking $\beta>0$ we have: if $\phi(r) \leq z_{2}=$ $\ln 8-2 \ln \left\{\sqrt{2} e^{-\beta / 2}-\left(2 e^{-\beta}-1\right)^{1 / 2}+\left(\sqrt{2} e^{-\beta / 2}+\left(2 e^{-\beta}-1\right)^{1 / 2}\right) r^{2}\right\}$ then $u$ tends to $w=\ln 8-2 \ln \left\{\sqrt{2}+1+(\sqrt{2}-1) r^{2}\right\}$ as $t$ tends to infinity, for all $\ln 2 \geq \beta \geq 0$. Again, we see that this is a new sufficient condition for global existence or, conversely, gives a necessary condition for blow-up. Again we note that near the boundary where $z=\beta$, we have $z_{2}>v \quad$ (= the nonminimal steady-state).

We show the arrangement for these bounds on Figure 2. The function $z_{1}$ provides an upper bound and $z_{2}$ a lower bound, since $\phi \geq z_{1}$ guarantees blow-up and $\phi \leq z_{2}$ guarantees global existence. Of course different values of $\beta$ can be chosen. We note that $z_{i}(0)$ and $z_{i}(1)$ are respectively monotonic decreasing and increasing with $\beta$, and so a pay-off for changing $\beta$ occurs. It should also be noted that the bounds $z_{i}(r)$ cross-over in the region and are on opposite sides of $w$ than what would be expected in the neighbourhood of $r=1$.

The same ideas can be used for comparing different boundary conditions if the problem is radially symmetric, or has different sized regions, provided

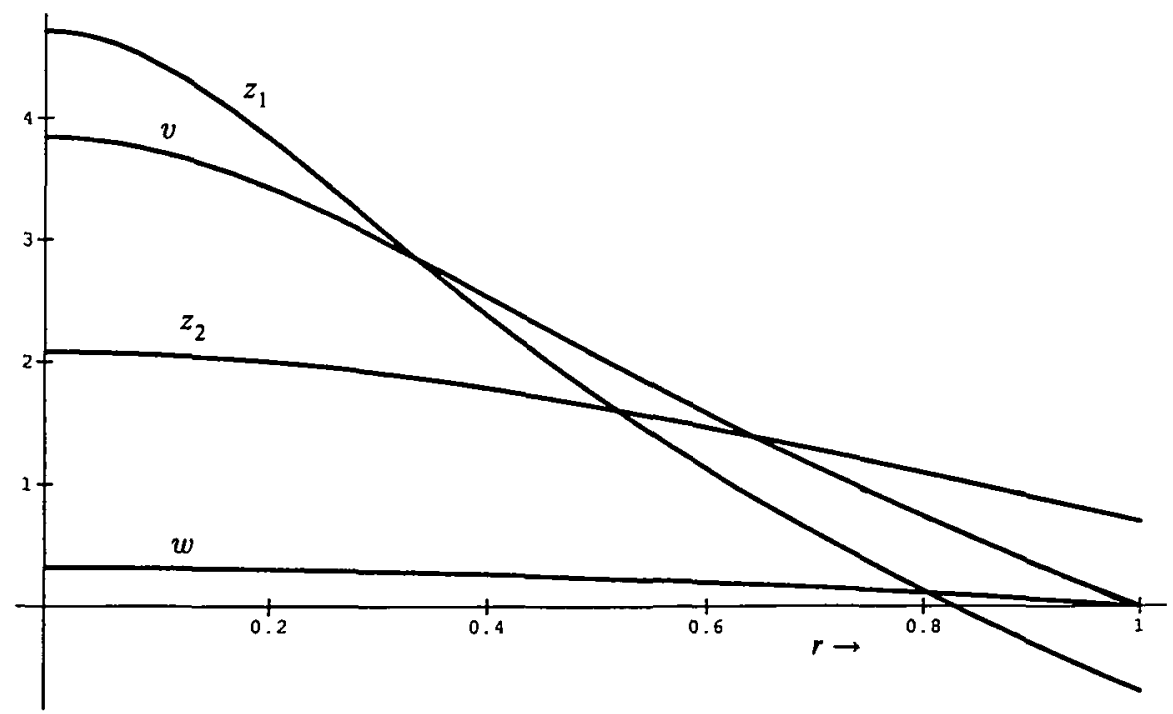

FIGURE 2. Illustrative arrangement for the upper $\left(z_{1}\right.$ with $\left.\beta=-\ln 2\right)$ and lower $\left(z_{2}\right.$ with $\beta=\ln 2$ ) bounds for critical initial data. 
the boundary conditions are Dirichlet $(\alpha=0)$. We get the following:

(A) If $\Omega$ is a ball and we define $B_{\beta} u$ by

$$
B_{\beta} u=a(\beta) u_{r}+(1-a(\beta)) u,
$$

with $a$ an increasing function of $\beta$; now taking $z$ to be a solution of

$$
\Delta z+f(z ; \beta)=0 \quad \text { in } \Omega ; \quad B_{\beta} z=g(\beta) \text { on } \partial \Omega ;
$$

then $\phi \leq z$ for $\beta \geq 0$ ensures a global solution while $\phi \geq z$ (with $z$ not the minimal solution) for $\beta \leq 0$ ensures blow-up.

(B) Take $\alpha=0$, that is, (2) becomes $u=0$ on $\partial \Omega$. If $\Omega \subset D$ and $z$ satisfies

$$
\Delta z+f(z ; \beta)=0 \text { in } D ; \quad z=g(\beta) \text { on } \partial D
$$

for $\beta \geq 0$, then $\phi \leq z$ in $\Omega$ ensures a global solution to (1), (2), (3). If $D \subset \Omega$ and $z$ is a nonminimal solution to

$$
\Delta z+f(z ; \beta)=0 \quad \text { in } D ; \quad z=g(\beta) \text { on } \partial D
$$

for $\beta \leq 0$ then $\phi \geq z$ in $D, \phi \geq 0$ in $\Omega \backslash D$ ensures that $u$ blows up.

\section{Structure of the set of critical initial data}

We may define two disjoint sets of initial data as follows

$$
\begin{aligned}
& \mathscr{A}=\{\phi: u \rightarrow w \text { as } t \rightarrow \infty\} \\
& \mathscr{B}=\{\phi: u \text { blows up completely }\}
\end{aligned}
$$

By the statement " $u$ blows up completely at some time $t_{c}$ " we mean that a solution (possibly a weak solution) exists for $t<t_{c}$ but not (even as a weak solution) for $t>t_{c}$. Equivalently if some approximate monotonic increasing regularising sequence of problems is considered for (1), (2), (3) their solutions tend to $u$ a.e. for $t<t_{c}$ and to infinity everywhere for $t>t_{c}$ (see Baras and Cohen [2], Lacey and Tzanetis [11]).

The critical conditions are those $\phi$ 's remaining (that is, not in $\mathscr{A} \cup \mathscr{B}$ ) :

$$
\begin{aligned}
& \mathscr{D}=(\phi: u \text { neither blows up completely nor tends to } w \text { as } t \rightarrow \infty) \\
& \mathscr{D}=\mathscr{A}^{\prime} \cap \mathscr{B}^{\prime} .
\end{aligned}
$$

We have the following properties:

(1) $\mathscr{A}$ and $\mathscr{B}$ are both open, so $\mathscr{D}$ is nonempty. The fact that $\mathscr{A}$ is open follows immediately from Lacey and Tzanetis [11]. The property for $\mathscr{B}$ needs further justification. Suppose $\phi \in \mathscr{B}$, then $u$ blows up completely 
at some $t=t_{c}$, that is, given a subregion $D$ of $\Omega, \bar{D} \subset \Omega$ the sequence of solutions $u_{n}$ to a regularised problem:

$$
\begin{gathered}
\partial u / \partial t-\Delta u=f_{n}(u), \quad x \in \Omega, \quad t>0, \\
B u=0, \quad x \in \partial \Omega, \quad t>0, \\
u(x, 0)=\phi(x), \quad x \in \Omega ;
\end{gathered}
$$

where, say,

$$
f_{n}(s)= \begin{cases}f(s), & s \leq n, \\ f(n), & s \geq n,\end{cases}
$$

satisfy, for $t=t_{1}>t_{c}, u_{n}(x, t) \rightarrow \infty$ uniformly for $x$ in $D$. But then given any $K$, there exists $\eta>0$, and $m$ such that the solution $u_{n}$ to

$$
\begin{gathered}
\partial u / \partial t-\Delta u=f_{n}(u), \quad x \in \Omega, \quad t>0, \\
B u=0, \quad x \in \partial \Omega, \quad t>0, \\
u(x, 0)=\phi(x)-\eta, \quad x \in \Omega,
\end{gathered}
$$

satisfies $u \geq K$ for $t=t_{t}, x \in D$, and $n \geq m$.

Applying method $C$ of Section 2 and noting that $\hat{u}_{n} \rightarrow \hat{u}-$ as $n \rightarrow \infty$ for $\hat{u}$ which is the solution to problem $(P)$ with $\phi-\eta$ replacing $\phi$, before any complete blow-up of $u$, we see that $u$ must blow up, that is, $\phi-\eta \in \mathscr{B}$. We conclude that if $|\phi-\tilde{\phi}|<\eta$ then $\tilde{\phi} \in \mathscr{B}$, that is $\mathscr{B}$ is open.

(2) Convexity. Using comparisons (upper and lower solutions) it is easy to show that:

if $\phi_{1}, \phi_{2} \in \mathscr{A}$ then $\beta \phi_{1}+(1-\beta) \phi_{2} \in \mathscr{A}$ for $0<\beta<1$,

if $\phi_{1}, \phi_{2} \in \mathscr{D}$ then $\beta \phi_{1}+(1-\beta) \phi_{2} \in\left\{\begin{array}{l}\mathscr{A} \cup \mathscr{D} \text { for } 0<\beta<1, \\ \mathscr{A} \cup \mathscr{B} \text { for } \beta<0 \text { or } \beta>1,\end{array}\right.$ if $\phi_{1}, \phi_{2} \in \mathscr{B}$ then $\beta \phi_{1}+(1-\beta) \phi_{2} \in \mathscr{B}$ for $\beta<0, \mathscr{B}>1$.

(3) Bounds (see Section 3)

If $\phi<z$ for $\beta \geq 0$ or $\phi \leq z$ for $\beta>0$ then $\phi \in \mathscr{A}$.

If $\phi>z$ for $\beta \leq 0$ or $\phi \geq z$ for $\beta>0$ then $\phi \in \mathscr{B}$.

(4) Ordering

If $\phi_{1} \in \mathscr{A}$ and $\phi_{2} \leq \phi_{1}$ then $\phi_{2} \in \mathscr{A}$.

If $\phi_{1} \in \mathscr{D}$ and $\phi_{2} \leq \phi_{1}$ then $\phi_{2} \in \mathscr{A} \cup \mathscr{D}$.

If $\phi_{1} \in \mathscr{D}$ and $\phi_{2} \geq \phi_{1}$ then $\phi_{2} \in \mathscr{B} \cup \mathscr{D}$.

If $\phi_{1} \in \mathscr{B}$ and $\phi_{2} \geq \phi_{1}$ then $\phi_{2} \in \mathscr{B}$.

(5) Comparison of regions (see Section 3)

Suppose $\phi_{1}$ is defined on $\Omega, \phi_{2}$ on $D$ with $\Omega \subset D$, with $\phi_{2} \geq \phi_{1}$ in $\Omega$, $\phi_{2} \geq 0$ in $D \backslash \Omega$. We obtain the following results by direct comparison of solutions of problem (1), (2), (3). 
If $\phi_{1} \in \mathscr{B}_{\Omega}$ then $\phi_{2} \in \mathscr{B}_{D}$.

If $\phi_{1} \in \mathscr{D}_{\Omega}$ then $\phi_{2} \in \mathscr{B}_{D} \cup \mathscr{D}_{n}$.

If $\phi_{2} \in \mathscr{D}_{D}$ then $\phi_{1} \in \mathscr{D}_{\Omega} \cup \mathscr{A}_{\Omega}$.

If $\phi_{2} \in \mathscr{A}_{D}$ then $\phi_{1} \in \mathscr{A}_{\Omega}$.

\section{Discussion}

We must end somewhat inconclusively, in that it is not always possible to determine what happens to $u$ if $\phi$ is critical (in $\mathscr{D}$ ). It is certainly possible that, if there is a steady-state solution $v$ other than the nonminimal one, then $u \rightarrow v$ as $t \rightarrow \infty$. We conjecture that this will invariably happen in one or two dimensions: if $\Omega \subseteq \mathbf{R}$ or $\Omega \subseteq \mathbf{R}^{2}$ then if $\phi \in \mathscr{D}, u \rightarrow v>w$ as $t \rightarrow \infty$. We see by the following argument that if $\phi_{1} \in \mathscr{D}$ and $\phi<\phi_{1}$ then $\phi \in \mathscr{A}$, that is $u \rightarrow w$ while if $\phi>\phi_{1}$, then $\phi \in \mathscr{B}$, that is $u$ blows up. Suppose that $\phi>\phi_{1}$. Define $z$ by

$$
\begin{array}{ccc}
\partial z / \partial t=\Delta z+f^{\prime}\left(u_{1}\right) z, & x \in \Omega, & t>0 ; \\
B z=0, & x \in \partial \Omega ; \\
z=\phi-\phi_{1} & \text { at } t=0 ;
\end{array}
$$

where $u_{1}$ is the solution of problem (1), (2), (3) with $u_{1}(x, 0)=\phi_{1}(x)$, and thus $z>0$. Also

$$
\frac{\partial}{\partial t}\left(u_{1}+z\right)-\Delta\left(u_{1}+z\right)-f\left(u_{1}+z\right)=f\left(u_{1}\right)+f^{\prime}\left(u_{1}\right) z-f\left(u_{1}+z\right) \leq 0
$$

since $f^{\prime \prime}>0$;

$$
\begin{array}{cl}
B\left(u_{1}+z\right)=0, & x \in \partial \Omega ; \\
u_{1}+z=\phi, & t=0 ;
\end{array}
$$

so $u_{1}+z$ is a lower solution for $u$ i.e. $u \geq u_{1}+z \geq z$. Also the principal eigenvalue of

$$
\begin{gathered}
\Delta \psi+f^{\prime}(v) \psi=\lambda \psi, \quad x \in \Omega, \\
B \psi=0, \quad x \in \partial \Omega
\end{gathered}
$$

has $\lambda_{1}>0$, and gives, since $u_{1} \rightarrow v, z \sim \psi_{1} e^{\lambda_{1} t}$ as $t \rightarrow \infty\left(\psi_{1}>0\right)$. Comparing this with method $D$ of Section 2 we see that $u$ must blow up completely.

For $\phi<\phi_{1}$ we clearly have $u<u_{1}$ so $u$ is bounded. It must then have an omega limit set, $\omega(\phi)$, with $\omega(\phi)$ a subset of the steady-states. But since $u<u_{1}$ and $u_{1} \rightarrow v$ the only possible steady-states in $\omega(\phi)$ are $v$ and $w$. Moreover if $v \in \omega(\phi)$ we would have a sequence $t_{n} \rightarrow \infty$ with $u\left(\cdot, t_{n}\right) \rightarrow v$ 
and we could deduce by similar reasoning to the above, that $u_{1}$ blows up. Thus $\omega(\phi)=\{w\}$ and $u \rightarrow w$ as $t \rightarrow \infty$.

We denote the set of $\phi$ such that $u \rightarrow v$ as $t \rightarrow \infty$ by $\mathscr{D}_{1}$. Certainly if $\phi_{1} \in \mathscr{D}_{1}$ then $\phi<\phi_{1}$ and $\phi \in \mathscr{A}$, that is, $u \rightarrow w$, while if $\phi>\phi_{1}$ then $\phi \in \mathscr{B}$ that is, $u$ blows up. A second possibility is that if $\phi \in \mathscr{D}$ then $u$ may exist in some weak sense for all time but may partially blow up at some time, that is, there exists $t^{*}<\infty$ such that $\|u\|_{\infty} \rightarrow \infty$ as $t \rightarrow t_{*}$ but $u$ exists for $t>t^{*}$. Such solutions are known to exist for $f(u)=e^{u}$ in dimension $N, 3 \leq N \leq 9$ (Lacey and Tzanetis [10]). We denote the set of $\phi$ leading to partial blow-up by $\mathscr{D}_{2}$. Again we have that for $\phi_{1} \in \mathscr{D}_{2}$ then $\phi<\phi_{1}$ has $\phi \in \mathscr{A}$, while $\phi>\phi_{1}$ has $\phi \in \mathscr{B}$. One final possibility is that there may exist $\phi \in \mathscr{D}$ such that $u$ exists classically for all time but $\|u\|_{\infty} \rightarrow \infty$ as $t \rightarrow \infty$. We denote the set of these initial conditions (if they exist) by $\mathscr{D}_{3}$. For $\phi=\phi_{3} \in \mathscr{D}_{3}$ then, at least for radially symmetric problems, $u \rightarrow w$ (the minimal steady state) as $t \rightarrow \infty$ except for $t=0$ while $u(0, t) \rightarrow \infty$. If $\phi>\phi_{3}$ it is possible to show (again for radially symmetric problems in $\mathbf{R}^{3}$ with $f(u)=e^{u}$ ) that $u$ blows up completely i.e. $\phi \in \mathscr{B}$ (Tzanetis [14]). We note that each of these comparisons give stronger results than the convexity and ordering results in (2), (4) of Section 4.

\section{Acknowledgement}

The award of a grant from the UK Sciences and Engineering Research Council (GR/D/73096) to one of the authors (GCW) during part of this work is gratefully acknowledged.

\section{References}

[1] J. Ball, "Remarks on blow-up and nonexistence theorems for nonlinear evolution equations", Quart. J. Maths. 28 (1977) 473-486.

[2] P. Baras and L. Cohen, "Complete blow-up after $T_{\max }$ for the solution of a semilinear heat equation", J. Func. Anal. 71 (1987) 142-174.

[3] H. Bellout, "A criterion for blow-up of solutions to semilinear heat equations", SIAM J. Math. Anal. 18 (1987) 722-727.

[4] T. Boddington, P. Gray, and G. C. Wake, "Criteria for thermal explosions with and without reactant consumption", Proc. Royal Soc. Lond. Series A 357 (1977) 403-422.

[5] H. Fujita, "On the nonlinear equation $\Delta u+e^{u}=0$ and $v_{t}=\Delta v+e^{v}$, Bull. Amer. Math. Soc. 75 (1965) 132-135.

[6] B. F. Gray and S. K. Scott, "The influence of initial temperature excess on critical conditions for thermal explosion", Combustion and Flame 61 (1985) 227-237. 
[7] S. Kaplan, "On the growth of solutions of quasilinear parabolic equations", Com. Pure Appl. Math. 16 (1963) 1361-1366.

[8] H. B. Keller and D. S. Cohen, "Some positone problems suggested by nonlinear heat generations”, J. Math. Mech. 16 (1967) 1361-1366.

[9] A. A. Lacey, "Mathematical analysis of theremal runaway for spatially inhomogeneous reactions", SIAMS J. Appl. Math. 43 (1983) 1350-1366.

[10] A. A. Lacey and D. E. Tzanetis, "Global unbounded solutions to a semilinear heat equation" (in preparation).

[11] A. A. Lacey and D. E. Tzanetis, "Complete blow-up for a semilinear diffusion equation with a sufficiently large initial condition", IMA J. of Applied Math. 41 (1988) 207-215.

[12] D. H. Sattinger, "Monotone methods in nonlinear elliptic and parabolic boundary value problems", Indiana Univ. Mat. J. 21 (1972) 979-1000.

[13] D. E. Tzanetis, "Global existence and asymptotic behaviour of unbounded solutions for the semilinear heat equation". Ph. D. thesis, Heriot-Watt Univ. Edinburgh 1986.

[14] D. E. Tzanetis, "Nearly convergent unbounded solutions for semilinear heat equations" (in preparation).

[15] G. C. Wake and M. E. Rayner, "Variational methods for nonlinear eigenvalues association with thermal ignition', J. Diff. Eqns. 13 (1973) 247-256. 